$62^{\text {ème }}$ Congrès de la SFCO, 02012 (2014)

DOI: $10.1051 /$ sfco/20146202012

(C) Owned by the authors, published by EDP Sciences, 2014

COMMUNICATION

\title{
Traitement par corticothérapie locale et laser diode à basse énergie des ulcérations buccales induites par les inhibiteurs de mTOR.
}

Pannérec V, Fricain JC

Pôle d'Odontologie et de Santé Buccale, CHU de Bordeaux

\section{Introduction}

Le Temsirolimus est un inhibiteur spécifique de mTOR (mammalian target of rapamycin) utilisé pour le traitement du carcinome rénal, du lymphome des cellules du manteau et plus récemment dans le cancer du sein métastatique. Des ulcérations buccales sont observées chez plus de $40 \%$ des sujets. Ces ulcérations peuvent être responsables d'une diminution de la posologie ou d'un arrêt du traitement. Aucun traitement de ces ulcérations n'a été rapporté.

\section{Observation}

Le premier cas décrit concernait une patiente traitée pour un cancer du sein métastatique, le deuxième un patient traité pour un carcinome rénal. Ils présentaient des ulcérations buccales aphtoïdes douloureuses, localisées sur la langue pour la première et sur la face interne de la lèvre pour le second. Ces lésions sont apparues 3 à 4 jours après la prise de Temsirolimus. La douleur générée par les ulcérations était cotée à 9 sur une échelle de 0 à 10 . Les ulcérations ont été traitées par application locale de Clobétazol/Xylocaine et biostimulation laser $\left(4 \mathrm{~J} / \mathrm{cm}^{2}\right)$. Immédiatement après la réalisation du laser les symptômes se sont atténués, la douleur était cotée à 3 . Quatre jours plus tard les ulcérations avaient complètement disparues.

\section{Discussion}

Le Clobétasol topique a démontré son efficacité dans la prise en charge des ulcérations buccales sous Sirolimus chez les patients transplantés du rein (Fricain.2008). Une méta-analyse témoigne de l'efficacité du traitement par la Low Level Laser Therapy (LLT) dans les mucites radio-chimio induites (Bensadoun 2012). L'adjonction du laser diode de basse énergie $(810 \mathrm{~nm})$ en respectant la fenêtre thérapeutique de 2 à $4 \mathrm{~J} / \mathrm{cm}^{2}$, au traitement par Clobétasol pourrait permettre d'optimiser l'effet antalgique (Sakurai 2000) et favoriser la cicatrisation (Schindl.2003).

\section{Conclusion}

La biostimulation par les lasers à basse énergie stimulerait la prolifération dans les tissus altérés tels que les cellules épithéliales (Dyson 1986), les cellules nerveuses (Bolton 1995), les fibroblastes (Nomura 2001) ou encore les cellules endothéliales (Schindl 2003), et aurait une influence positive au niveau des médiateurs de l'inflammation (Yu 1994).

This is an Open Access article distributed under the terms of the Creative Commons Attribution License 4.0, which permits unrestricted use, distribution, and reproduction in any medium, provided the original work is properly cited. 